\title{
Efeitos do butorfanol e da morfina em pombos domésticos (Columba livia) anestesiad os com isofluorano e submetidos à cirurgia ortopédica*
}

\author{
Effects of butorphanol and mophine in pigeons (Columba livia) \\ anaesthetised with isofluorone on orthopeadic surgery \\ Luciana Dambrósio Guimarães ${ }^{1}$, Marcelo Meller Alievi², Alceu Gaspar Raiser ${ }^{3}$, \\ Fabíola Niederauer Flores ${ }^{4}$, Simone Bopp ${ }^{5}$, Patrícia Almeida Ferreira ${ }^{3} \&$ Carolina Traesel $^{3}$
}

\begin{abstract}
RESUMO
A anestesia balanceada é muito utilizada na Medicina Veterinária, porém em aves, muitos animais ainda são anestesiados somente com anestésico inalatório devido às divergências a respeito do uso de opióides. Objetivou-se avaliar os efeitos cardiovasculares e respiratórios produzidos por opióides, associados ao isofluorano, em pombos domésticos submetidos à osteotomia e osteossíntese de úmero. Para isso foram utilizados 12 animais clinicamente sadios, submetidos à medicação pré-anestésica com $3 \mathrm{mg} / \mathrm{kg}$ de butorfanol (B) ou 3mg/kg de morfina (M) e anestesiados com 1,5\% de isofluorano. A freqüência cardíaca (FC), a freqüência respiratória (f) e pressão arterial sistólica (PAS) foram mensuradas antes da administração dos opióides (A), durante a divulsão dos tecidos (D), imediatamente após a osteossíntese $(\mathrm{O})$ e na redução do espaço morto (EM). Em A e ao final do procedimento cirúrgico (F) foi mensurada a temperatura clocacal (TC) e foi realizada a colheita de sangue arterial para hemogasometria. No pós-operatório imediato foi avaliado o tempo até a extubação (TE). Pode-se observar que o grupo B apresentou valores inferiores de FC em relação aos basais e ao grupo M, enquanto que a PAS foi inferior somente quando comparada aos valores basais, no grupo B. A $\mathrm{SpO}_{2}$ aumentou e a TC reduziu no período de manutenção anestésica, sem diferença estatística entre os grupos. Houve leve acidose respiratória no grupo B, identificada pela redução do $\mathrm{pH}$ em relação aos valores basais. Não foi observada diferença significativa no TE entre os grupos. Pode-se concluir que a morfina ou o butorfanol associados ao isofluorano podem ser utilizados com segurança em pombos domésticos, por produzirem mínimos efeitos sobre o sistema cardiovascular e respiratório.
\end{abstract}

Descritores: opióides, aves, anestesia.

\section{ABSTRACT}

A balanced anesthesia is common used in Veterinary Medicine, however, due different points of view in respect to opioid affects in birds, they still being anesthetized only with inalatory gases. The aim of this work was to evaluate the cardiovascular and respiratory effects on isofluorane associated anesthesia in pigeons submitted to humerus ostectomy and repair. For that 12 clinically heath animals were used under the following protocol: $3 \mathrm{mg} / \mathrm{kg}$ de butophanol (B) or $3 \mathrm{mg} / \mathrm{kg}$ de morphine (M) as a pre-anaesthetic and anesthetized with isofluorane 1,5\%. A heart frequency (FC), respiratoty frequency (F) and arterial blood pressure (PAS) were measured before the opiod injection (A), during the humerus approach (D), immediate after the bone be repaired $(\mathrm{O})$ and suture of dead space $(\mathrm{EM})$. The cloacal temperature was taken at the beginning and the end of the procedure and arterial blood sample was taken to gasometric analysis (time points A and F). Soon after the procedure the time for extubation (TE) was observed. The group B presented lowers values in relation to basal levels and group M, however PAS was lower only compared to basal levels on group $\mathrm{B}$. The $\mathrm{SpO} 2$ increased and TC decreased during the period of anesthetic maintenance without any difference between groups. There was mild respiratory acidosis on group B, identified by decrease on $\mathrm{pH}$ in relation to basal levels. There was not difference on TE between groups. We could conclude that morphine or butorphanol associated with isofluorane produce minimal effects $\mathrm{n}$ cardiac and respiratory systems and can be securely used in pigeons.

Key words: opioids, birds, anaesthesia. (UFMT), Cuiabá, MT/Brasil. ${ }^{2}$ Universidade Federal do Rio Grande do Sul (UFRGS), Porto Alegre, RS/Brasil. ${ }^{3}$ UFSM. ${ }^{4}$ Universidade do Estado de Santa Catarina (UDESC), Lages, SC/Brasil. ${ }^{5}$ Faculdade Integrado de Campo Mourão, Campo Mourão, PR/Brasil. CORRESPONDÊNCIA: L.D. Guimarães [ldguimaraes@ufmt.br ; Fax 65 36158614]. 


\section{INTRODUÇÃO}

A ocorrência de fraturas no úmero de aves é freqüente e as respostas comportamentais que identificam os sinais de dor são difíceis de perceber [11]. Estudos e experiências clínicas sugerem que a nocicepção em aves é mediada por vias neurais e neurotransmissores similares aos de mamíferos, o que confirma que essa classe animal é capaz de perceber a dor $[1,5,12]$.

Estudos identificaram maior porcentagem de receptores kappa em relação aos mu no cérebro de pombos domésticos. Esses dados foram extrapolados para as outras espécies de aves sugerindo que os fármacos agonistas dos receptores mu, como a morfina, seriam menos eficiente do que os agonistas dos receptores kappa, como o butorfanol, na produção de analgesia [12-14]. Os estudos com a morfina são contraditórios, porém foi observado que tanto a morfina quanto o U50488H, um kappa agonista, promoveram a redução da Concentração Alveolar Mínima (CAM) em galinhas anestesiadas com isofluorano, o que indica potencial analgésico ou sedativo e sugere que tanto receptores mu quanto kappa estão presentes nessa espécie e que ambos agem de maneira similar [11].

A analgesia preemptiva apresenta as vantagens de produzir anestesia balanceada e analgesia [7,9,11]. É necessário esclarecer se os opióides administrados no período pré-operatório produzem efeitos adversos nos sistemas cardiovascular e respiratório durante o trans-operatório ou prolongam o tempo de recuperação anestésica [9,11]. O objetivo desse estudo é avaliar os efeitos cardiovasculares e respiratórios produzidos pela morfina e pelo butorfanol, associados ao isofluorano, em pombos domésticos (Columba lívia) submetidos à osteotomia e osteossíntese de úmero.

\section{MATERIAIS E MÉTODOS}

Foram utilizados 12 pombos domésticos ( $\mathrm{Co}$ lumba livia), com peso médio de 358 gramas, considerados clinicamente sadios após exame físico e submetidos a um período de adaptação em gaiolas individuais por 15 dias. Os animais foram divididos aleatoriamente em dois grupos. No grupo $\mathrm{M}$ foi utilizado como medicação pré-anestésica $3 \mathrm{mg} / \mathrm{kg}$ de morfina e no grupo $\mathrm{B}, 3 \mathrm{mg} / \mathrm{kg}$ de butorfanol, ambos administrados pela via intramuscular (IM), na musculatura peitoral, 20 minutos antes da indução anestésica com isofluo- rano. A indução anestésica foi realizada com máscara facial conectada ao sistema não reinalatório de Bain, com $4,5 \%$ de isofluorano, administrado através de vaporizador calibrado, e fluxo diluente de $1 \mathrm{~L} / \mathrm{min}$. de $\mathrm{O}_{2}$ a $100 \%$. Após a perda do reflexo palpebral e relaxamento das asas os animais foram intubados com sonda orotraqueal de murphy número 2 a 3,5, dependendo do tamanho do animal, sem balonete. Os pombos foram posicionados em decúbito esternal sobre bolsas com água aquecida, de maneira a manter a temperatura corporal acima de $38^{\circ} \mathrm{C}$, durante todo o procedimento cirúrgico cuja duração média foi de 30 minutos.

Os animais foram anestesiados para a remoção das penas da área do campo cirúrgico e antissepsia. A concentração de isofluorano inicial, determinada pelo vaporizador calibrado, foi de $1,5 \%$. A mesma foi aumentada em $0,5 \%$ quando os animais manifestaram resposta motora desencadeada pelo estímulo cirúrgico.

O procedimento cirúrgico constou de uma incisão na face dorsal da porção diafisária do úmero direito, divulsão do tecido subcutâneo e afastamento da musculatura da região e do nervo radial. A osteotomia foi realizada com uma serra oscilatória e a osteossíntese com a associação de dois pinos intramedulares e acrílico autopolimerizável estéril, inserido no canal medular da porção distal da fratura. A seguir foi realizado a redução de espaço morto e sutura de pele.

No período pós-operatório imediato os animais foram envoltos em toalhas de papel para evitar lesões no membro operado, na tentativa de se posicionar em posição bipedal. Para analgesia pós-operatória foi utilizado flunixim meglumine na dose de $5 \mathrm{mg} / \mathrm{kg}$ IM durante 3 dias.

\section{Períodos de avaliação e variáveis mensuradas}

As variáveis frequiência cardíaca (FC), frequiência respiratória (f) e pressão arterial sistólica (PAS) foram mensuradas antes da administração dos opióides (A), durante a divulsão dos tecidos (D), imediatamente após a osteossíntese $(\mathrm{O})$ e na redução do espaço morto (EM). Em A e ao final do procedimento cirúrgico $(\mathrm{F})$ foi mensurada a temperatura clocacal (TC) e foi realizada a colheita de sangue arterial para hemogasometria. $\mathrm{O}$ sangue foi colhido através de punção direta das artérias braquial ou ulnar. A concentração de isofluorano liberada pelo vaporizador 
(C) foi registrada durante o período trans-operatório. No pós-operatório imediato foi avaliado o tempo até a extubação (TE).

A FC foi mensurada através de oxímetro de pulso, a $\mathrm{f}$ através do movimento da parede costal, a PAS através de método não invasivo com Doppler ultrassônico e a TC através de termômetro digital. Os gases sangüíneos e bicarbonato foram avaliados através de analisador de gases sangüíneos a $37^{\circ} \mathrm{C}$.

Os dados foram comparados através da análise de variância (ANOVA) e teste de Tukey utilizando o softhware Graph Pad Instant ${ }^{\circledast}$.

\section{RESULTADOS}

O grupo B apresentou valores inferiores de FC quando comparados aos valores basais $(p<0,05)$ e ao grupo M em D (p <0,03), O (p <0,0001) e EM (p $<0,0001)$. A PAS foi inferior aos valores basais no grupo $\mathrm{B}$, em todos os tempos ( $\mathrm{p}<0,05)$, enquanto que no grupo $\mathrm{M}$ foi inferior ao basal somente em $\mathrm{D}$ ( $p<0,05)$, sem diferença entre os grupos. A $\mathrm{SpO}_{2}$ aumentou em ambos os grupos em todos os tempos ( $p$ $<0,05)$, sem diferença entre os grupos. Não houve diferença significativa na concentração de isofluorano utilizada entre os grupos ou tempos (Tabela 1). Não pode-se estabelecer valores confiáveis de $f$ durante o período trans-operatório.

No final da cirurgia, o valor de $\mathrm{pH}$ foi significativamente inferior no grupo B ( $\mathrm{p}<0,0061)$ quando comparado aos valores basais, sem diferença entre $\mathrm{M}$ e B. Não foram observadas alterações na $\mathrm{PaCO}_{2} \mathrm{e}$ bicarbonato entre os grupos ou tempos (Tabela 2).

Tabela 1. Valores médios e desvio padrão das variáveis freqüência cardíaca (FC), pressão arterial sistólica (PAS), saturação de oxigênio na hemoglobina $\left(\mathrm{SpO}_{2}\right)$ e concentração de isofluorano $(\mathrm{C})$, em pombos domésticos pré-medicados com butorfanol (B) ou morfina (M) e anestesiados com isofluorano para realização de cirurgia ortopédica. Os dados foram obtidos antes da pré-medicação (BA), durante a divulsão dos tecidos (D), logo após a osteossíntese (O) e na redução de espaço morto (EM).

\begin{tabular}{|c|c|c|c|c|c|}
\hline Variáveis & Grupo & BA & D & 0 & EM \\
\hline \multirow{2}{*}{ FC (bpm) } & $M$ & $168,20 \pm 14,70$ & $133,33 \pm 25,86$ & $148,33 \pm 46,00$ & $154,20 \pm 18,77$ \\
\hline & B & $167,17 \pm 37,06$ & $121,33 \pm 13,68^{*}$ & $125,33 \pm 12,82^{*}$ & $121,6 \pm 09,53^{\star *}$ \\
\hline \multirow{2}{*}{ PAS $(\mathrm{mmHg})$} & $M$ & $152,67 \pm 28,46$ & $099,33 \pm 33,72^{*}$ & $105,50 \pm 22,78$ & $108,20 \pm 33,06$ \\
\hline & B & $136,33 \pm 18,99$ & $085,00 \pm 20,46^{*}$ & $087,67 \pm 22,81^{*}$ & $085,60 \pm 20,95^{\star}$ \\
\hline \multirow{2}{*}{$\mathrm{SpO}_{2}(\%)$} & $M$ & $94,35 \pm 03,02$ & $99,70 \pm 0,32^{*}$ & $99,80 \pm 0,02^{*}$ & $99,80 \pm 0,37^{*}$ \\
\hline & B & $94,82 \pm 01,08$ & $99,30 \pm 0,25^{*}$ & $99,60 \pm 1,26^{*}$ & $99,32 \pm 0,67^{\star}$ \\
\hline \multirow{2}{*}{$\mathrm{C}(\%)$} & $\mathbf{M}$ & - & $1,58 \pm 0,20$ & $1,67 \pm 0,40$ & $1,58 \pm 0,20$ \\
\hline & B & - & $1,50 \pm 0,00$ & $1,67 \pm 0,26$ & $1,67 \pm 0,22$ \\
\hline
\end{tabular}

"Diferença significativa entre os tempos em relação aos valores basal $(p<0,05)$. Diferença significativa entre os grupos $(p<0,05)$.

Tabela 2. Valores médios e desvio padrão das pH, concentração parcial arterial de dióxido de carbono $\left(\mathrm{PaCO}_{2}\right)$ e bicarbonato, em pombos domésticos pré-medicados com butorfanol (B) ou morfina (M) e anestesiados com isofluorano para realização de cirurgia ortopédica. Os dados foram obtidos antes da pré-medicaçãono final do procedimento cirúrgico $(\mathrm{F})$.

\begin{tabular}{cccc}
\hline Variáveis & Grupo & BA & F \\
\hline $\mathrm{pH}$ (unidades) & $\mathrm{M}$ & $7,36 \pm 0,07$ & $7,31 \pm 0,05$ \\
& $\mathrm{~B}$ & $7,34 \pm 0,03$ & $7,28 \pm 0,03^{*}$ \\
\hline $\mathrm{PaCO}_{2}(\mathrm{mmHg})$ & $\mathrm{M}$ & $32,83 \pm 12,48$ & $47,22 \pm 13,50$ \\
& $\mathrm{~B}$ & $36,16 \pm 03,13$ & $47,3112,48$ \\
\hline $\mathrm{HCO}_{3}(\mathrm{mmol} / \mathrm{l})$ & $\mathrm{M}$ & $17,40 \pm 04,89$ & $22,47 \pm 04,42$ \\
& B & $19,25 \pm 02,12$ & $21,55 \pm 06,27$ \\
\hline
\end{tabular}

"Diferença significativa entre os tempos em relação aos valores basal $(\mathrm{p}<0,05)$. 
No pós-operatório imediato pode-se observar

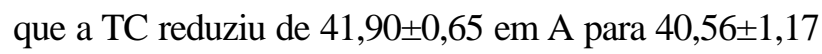
( $\mathrm{p}<0,03$ ) em $\mathrm{F}$ no grupo $\mathrm{M}$ e de 41,97 $\pm 0,51$ para $40,12 \pm 0,72$ ( $p<0,0004)$ no grupo $B$, sem diferença estatística entre os grupos. Não houve diferença significativa no TE entre os grupos, que foi de 7,1 $\pm 3,33$ no grupo $\mathrm{M}$ e 4,78 $\pm 1,72$ no grupo $\mathrm{B}$.

\section{DISCUSSÃO}

As aves representam a classe de maior número entre os vertebrados, com aproximadamente 9.700 espécies. Embora a literatura cite vários analgésicos e doses para aves, os estudos que comprovam seus efeitos são muito poucos. Muitos animais ainda são anestesiados somente com anestésicos inalatórios (isofluorano ou sevofluorano), que promovem depressão do sistema nervoso central suficiente para prevenir a percepção da dor durante a cirurgia, mas não produzem analgesia, podendo haver ainda depressão excessiva do sistema cardiovascular e respiratório. [6,11].

Os opióides podem produzir analgesia em muitas espécies de animais exóticos, mas os efeitos podem variar de acordo com a espécie e em indíviduos da mesma espécie e, por isso, os resultados obtidos são controversos [7,13]. Os efeitos adversos produzidos pelos opióides e que são comumente observados são bradicardia e depressão respiratória [7].

O butorfanol é um opióide agonista dos receptores kappa e antagonista dos receptores mu muito utilizado na Medicina Veterinária e produz analgesia dose-dependente com menor depressão respiratória do que a morfina, em várias espécies de animais domésticos [7,11,15]. A dose recomendada para aves varia entre 1 e $3 \mathrm{mg} / \mathrm{kg}$ pela via intramuscular [9].

No presente estudo, os valores de frequiência respiratória não foram exatos devido à dificuldade de visualização da parede costal, já que os animais permaneceram em decúbito esternal com as asas sobre o tórax e cobertos por panos de campos e, por isso, os dados não foram incluídos na análise estatística. A $\mathrm{SpO}_{2}$ manteve-se com valores acima dos basais devido ao fornecimento de $\mathrm{O}_{2}$ a $100 \%$ e isso também sugere que a ventilação espontânea foi adequada, o que foi observado em papagaio de hispaniola (Amazona ventralis) [9].

Em cacatuas, $1 \mathrm{mg} / \mathrm{kg}$ de butorfanol associado ao isofluorano não alterou de maneira significativa a f [3,9]. Em papagaio de hispaniola anestesiados com sevofluorano e submetidos a endoscopia para avaliação das gônadas, o butorfanol, na dose de $2 \mathrm{mg} / \mathrm{kg}$ IM, administrado 20 minutos antes da indução anestésica, produziu redução na $f$ e aumento na concentração final de $\mathrm{CO}_{2}$ expirado, mas os efeitos foram atribuídos ao sevofluorano [9].

Pode-se observar que o butorfanol produziu leve acidose respiratória, evidenciado pelo menor valor de $\mathrm{pH}$ em relação aos valores basais, no entanto não foi observada diferença entre os efeitos produzidos pela morfina. Ambos os grupos produziram leve hipercapnia já que a $\mathrm{PaCO}_{2}$ apresentou-se acima de $45 \mathrm{mmHg}$.

Em relação ao sistema cardiovascular, o butorfanol associado ao isofluorano produziu redução na FC quando comparado aos valores basais, o que também foi observado em cacatuas e papagaio de hispaniola [3,9]. Em mamíferos, os opióides aumentam o tono vagal, associado com bradicardia, e um mecanismo de ação similar pode ocorrer em aves [3]. No entanto, a redução ocorrida neste estudo não foi considerada bradicardia. Outros pesquisadores utilizaram $0,1 \mathrm{mg} / \mathrm{kg}$ de butorfanol em perus anestesiados com halotano e observaram que não houve diferença em relação ao controle nas variáveis FC, f e concentração de halotano liberada pelo vaporizador [15].

Em cacatuas e galinhas, a pressão arterial não foi alterada quando utilizado butorfanol associado ao isofluorano, no entanto, neste estudo em pombos domésticos foi observado redução da PAS em relação aos valores basais. [2,3].

Comparando-se os efeitos da morfina e do butorfanol pode-se observar que o segundo produziu valores inferiores de FC. Em galinhas, a morfina e o U50488H foram utilizados nas doses de $0,1,1$ e $3 \mathrm{mg} /$ $\mathrm{kg}$, administrados pela via intravenosa, e não houve diferença estatística entre os grupos [2]. Não existem estudos comparando os efeitos da morfina e do butorfanol sobre o sistema cardiovascular e respiratório de aves. A morfina é pouco utilizada devido à contradições relacionadas com a dose, efeitos e a possibilidade de ineficácia pelo fato dessas espécies possuírem maior número de receptores kappa [7,12].

Em relação ao pós-operatório imediato, não houve diferença entre os grupos no tempo de extubação, considerado seguro para a espécie. Apesar da TC ter apresentado diferença significativa em relação aos valores basais, no final do procedimento cirúrgico, a bolsa com água quente foi eficaz em mantê-la acima 
dos $38^{\circ} \mathrm{C}$. Sabe-se que a hipotermia é uma das principais causas de mortalidade em aves [10].

Quando se associam fármacos anestésicos o efeito da interação pode ser positivo ou negativo e deve ser considerado no momento da escolha do protocolo anestésico [2]. Tanto a morfina quanto o butorfanol podem ser utilizados associados ao isofluorano na anestesia de pombos domésticos, pois promoveram alterações mínimas sobre o sistema cardiovascular e respiratório. A técnica de anestesia balanceada utiliza comumente a associação de anestésicos com opióides, administrados de maneira preemptiva, e podem produzir poucos efeitos adversos em aves, no entanto, são necessárias mais pesquisas na área [11].

Para reconhecer a dor em pacientes exóticos é preciso conhecer o comportamento normal da espécie e do indivíduo avaliado, além de variáveis fisiológicas como f, pressão arterial (PA), FC e níveis de catecolaminas e corticosteróides, que podem ser alterados pela dor ou estresse [7,12]. Baseado na redução da FC e f em relação aos valores basais, em papagaio de hispaniola, pôde-se sugerir que o butorfanol administrado antes da indução anestésica produziu analgesia [9]. No entanto, parece que em relação ao sistema cardiovascular e respiratório o melhor indicador de dor é a alteração da PA [7,12,13].

Considerando os resultados observados poderia-se dizer que tanto a morfina quanto o butorfanol foram eficientes na produção de analgesia para osteotomia e osteossíntese de úmero em pombos, já que as variáveis avaliadas, incluindo a PA, mantiveram-se abaixo dos valores basais durante os estímulos dolorosos produzidos pela cirurgia.

Existe uma raridade de informações na literatura científica sobre o uso de analgésicos em aves, além de contradição em relação aos seus efeitos. Pesquisas clínicas e informações sobre o sucesso ou dificuldades em relação aos tratamentos com analgésicos devem ser relatados na literatura veterinária, para expandir o conhecimento na área [11].

\section{CONCLUSÕES}

A morfina e o butorfanol podem ser utilizados com segurança associados ao isofluorano para anestesia de pombos domésticos submetidos à cirurgia ortopédica, pois produzem mínimos efeitos sobre os sistemas cardiovascular e respiratório.

Agradecimentos. Este trabalho teve suporte financeiro do CNPq e Hospital Veterinário da UFSM.

\section{REFERÊNCIAS}

1 Clyde V.L. \& Paul-Murphy J. 2000. Avian analgesia. In: Bonagura J.D. \& Kersey R. (Eds). Kirk's Current Veterinary Therapy: Small Animal Practice. 13th edn. Philadelphia: Saunders, pp.1126-1128.

2 Concannon K.T., Dodam J.R. \& Hellyer P.W. 1995. Influence of a mu- and kappa-opioid agonist on isoflurane minimal anesthetic concentration in chickens. American Journal Veterinary Research. 56: 806-810.

3 Curro T.G., Brunson D.B. \& Paul-Murphy J. 1994. Determination of ED50 of isoflurane and evaluation of the isofluranesparing effect of butorphanol in cockatoos (Cacatua spp.). Veterinary Surgery. 23: 429-433.

4 Dohoo S.E. 1990. Isoflurane as an inhalational anesthetic agent in clinical practice. Canadian Veterinary Journal. 31 : 847-850.

5 Gentle M.J. 1992. Pain in birds. Animal Welfare. 1: 235-247.

6 Harrison G.L. 1986. Response to avian sedation avaluation. Journal of Americam Veterinary Medicine Association. $209: 1992$.

7 Hawkins M. 2006. The use of analgesics in birds, reptiles, and small exotic mammals. Journal of Exotic Pet Medicine. 15: 177-192.

8 Hoopes S., Flammer K., Hoersch K., Papich M. \& Paul-Murphy J. 2003. Disposition and analgesic effects of fentanyl in white cockatoos (Cacatua alba). Journal of Avian Medicine and Surger. 17: 124-130.

9 Klaphake E., Schumacher J., Greenacre C., Jones M.P. \& Zagaya N. 2006. Comparative anesthetic and cardiopulmonary effects of pre- versus postoperative butorphnaol administration in hispaniolan amazon parrots (Amazona ventralis) anesthetized with sevoflurane. Journal of Avian Medicine and Surgery. 20: 2-7.

10 Korbel R. 1998. Comparative investigations on inhalation anesthesia with isoflurane (Forene) and sevoflurane (SEVOrane) in racing pigeons (Columba livia Geml.1789, var. domestica) and presentation of a reference anesthesia protocol for birds. Tieräztl Prax. 26: 211-223.

11 Paul-Murphy J., Brunson B. \& Miletic V. 1999. Analgesic effects of butorphanol and buprenorphine in conscious African grey parrots (Psittacus erithacus erithacus and Psittacus erithacus timneh). American Journal of Veterinary. Research. 60 : 1218-1221. 
12 Paul-Murphy J. \& Fialkowski J. 2001. Injectable anesthesia and analgesia of birds. Online. Disponível em: <http://www.ivis.org/ authorforms/contributor_info.asp?ID=450>. Acessado em 08/2001.

13 Paul-Murphy J. \& Ludders J.W. 2001. Avian analgesia. Veterinary Clinics of North America: Exotic Animal Practice. 4 : 35-45.

14 Machin K.L. 2005. Avian analgesia. Seminars in Avian and Exotic Pet Medicine. 14: 236-242.

15 Reim D.A. \& Middleton C.C. 1995. Use of butorphanol as an anesthetic adjunct in turkeys. Laboratory Animal Science. 45: 696-698.

16 Trim C.M. 1983. Cardiopulmonary effects of buthorphanol tartrate on dogs. American Journal of Veterinary Research. 44: 329-331.

17 Tyner C.L., Greene S.A. \& Harstfield S.M. 1989. Cardiovascular effects of butorphanol administration in isoflurane-O anesthetized healthy dogs. American Journal of Veterinary Research. 50: 1340-1342. 